\title{
CONVERGENCE OF THE TWO-PHASE STEFAN PROBLEM TO THE ONE-PHASE PROBLEM
}

\author{
BY \\ BARBARA E. STOTH \\ Department of Mathematics, Carnegie Mellon University, Pittsburgh, PA \\ Institut für Angewandte Mathematik, Universität Bonn, Germany
}

\begin{abstract}
We study the limit of the one-dimensional Stefan problem as the diffusivity coefficient of the solid phase approaches zero. We derive a weak formulation of the equilibrium condition for the resulting one-phase problem that allows jumps of the temperature across the interface. The weak formulation consists of a regularity condition that only enforces the usual equilibrium condition to hold from the liquid phase.

At the end we briefly discuss the radial problem in higher space dimensions.

The main tools in order to prove the convergence are the uniform bounds on the total variation of the free boundary that are derived using a regularized problem, where the equilibrium condition is substituted by a dynamical condition.
\end{abstract}

Introduction. The classical two-phase Stefan problem (in one space dimension) reads:

$$
\begin{aligned}
& u_{t}-c_{-} u^{\prime \prime}=0, \quad \text { if } 0<x<s(t), \\
& u_{t}-c_{+} u^{\prime \prime}=0, \quad \text { if } s(t)<x<a
\end{aligned}
$$

with no-flux conditions on the fixed boundary

$$
u^{\prime}(t, 0)=u^{\prime}(t, a)=0
$$

and together with the latent heat equation (or Stefan condition)

$$
\left(c_{+}\left(u^{+}\right)^{\prime}-c_{-}\left(u^{-}\right)^{\prime}\right)(t, s(t))=-l s_{t}(t)
$$

and the equilibrium condition

$$
u(t, s(t))=0
$$

Here $u$ is interpreted as the temperature deviation from the melting temperature, the set $\Gamma:=\{x=s(t)\}$ represents the interface separating the liquid $(x>s(t))$ and

Partially supported by the Army Research Office through the Center for Nonlinear Analysis. 
solid $(x<s(t))$ phases, $\left(u^{+/-}\right)^{\prime}$ are the limits of $u^{\prime}$ from the corresponding side of the interface, $l$ is the (positive) latent heat and $c_{-}$and $c_{+}$are the (positive) heat diffusivity coefficients of the two phases (resp.). The system is completed by initial conditions $u_{0}$ for the temperature and $s_{0}$ for the location of the free boundary. We stress that we do not impose any sign condition for the initial temperature, so that superheating and supercooling effects are included.

The objective of this paper is to study the behaviour of a particular class of solutions of the two-phase Stefan problem as one of the diffusivity coefficients approaches zero.

To this end we set $c_{-}=\delta$ and $c_{+}=1$. Thus we are interested in the limit $\delta \rightarrow 0$. Formally, we expect the one-phase problem

$$
\begin{aligned}
u_{t}=0, & \text { if } 0<x<s(t), \\
u_{t}-u^{\prime \prime}=0, & \text { if } s(t)<x<a,
\end{aligned}
$$

with no-flux conditions on the fixed boundary

$$
u^{\prime}(t, 0)=u^{\prime}(t, a)=0
$$

the latent heat condition

$$
\left(u^{+}\right)^{\prime}(t, s(t))=-l s_{t}(t)
$$

and the equilibrium condition

$$
u(t, s(t))=0 \text {. }
$$

But, in particular in the case of overheated initial data (so that the free boundary moves into the solid region), the equilibrium condition contradicts the differential equation $u_{t}=0$ in the solid phase, if the initial condition in the solid phase is different from $\equiv 0$, resulting in a jump of the temperature $u$ across the free boundary.

In order to overcome this difficulty we will introduce a weak formulation of the onephase Stefan problem (see Definition 1.1 in Sec. 1) where the equilibrium condition is substituted by an integrability condition. We let

$$
\varphi(t, x):=H(x-s(t))
$$

given in terms of the Heaviside function $H$, be the characteristic function of the liquid phase. Then we ask that

$$
u \varphi \in L^{2}\left(0, T ; H^{1,2}(0, a)\right)
$$

This replaces the equilibrium condition in the following sense. By definition of $\varphi$,

$$
(u \varphi)(t, x)= \begin{cases}0, & \text { if } x<s(t), \\ u^{+}(t, x), & \text { if } x>s(t) .\end{cases}
$$

Since this function has to be in $H^{1.2}(0, a)$ for almost all $t$, it has to be continuous and thus

$$
u^{+}(t, s(t))=0 \quad \text { for almost all } t \in(0, T)
$$


Thus the original equilibrium condition is satisfied for the limit from the "liquid" phase. In the solid phase the initial values of the temperature $u$ are just transported, so that according to the initial condition there might be a jump on the temperature across the interface.

In Sec. 1 we will prove that a particular class of solutions of the two-phase problem converges to such a solution of the one-phase problem, as the diffusivity coefficient $\delta$ approaches zero (cf. Theorem 1.9).

We still have to make precise what we mean by "particular" in the above. We only consider solutions of the two-phase problem that can be obtained through a regularization process: we assume that they may be approximated by a sequence of solutions of a regularized Stefan problem, where the equilibrium condition is substituted by a dynamical condition, namely

$$
u(t, s(t))=-\alpha s(t) .
$$

In Sec. 1 we give the precise definitions of the two-phase Stefan and regularized Stefan problem, thereby following the approach of Visintin (1987), as well as the definition of the one-phase problem. Then we state the basic estimates (Proposition 1.5) and prove the main theorem (Theorem 1.9) that establishes the connection between the two-phase and one-phase problems.

In Sec. 2 we prove Proposition 1.5, which contains the basic mathematics of our problem.

In Sec. 3 we briefly discuss the radial problem. It turns out that an equivalent of Proposition 1.5 holds true, such that basically the same method as in the one-dimensional problem applies.

Section 1. The one-dimensional problem. We start giving the precise notion of a solution of the one-phase Stefan problem.

Definition 1.1. (One-phase Stefan problem) A pair of functions $u \in L^{\infty}(0, T$; $\left.L^{2}(0, a)\right)$ and $s \in \operatorname{BV}(0, T)$ with values in $[0, a]$ is a solution of the one-phase Stefan problem with initial condition $u_{0} \in L^{2}(0, a)$ and $s_{0} \in[0, a]$ if for the order parameter

$$
\varphi(t, x):=H(x-s(t)), \quad \varphi_{0}(x):=H\left(x-s_{0}\right)
$$

given in terms of the Heaviside function $H$ and the temperature $u$, the equation

$$
-\int_{0}^{T} \int_{0}^{a}(u+\varphi) v_{t} d x d t-\int_{0}^{a}\left(u_{0}+\varphi_{0}\right)(x) v(0, x) d x-\int_{0}^{T} \int_{0}^{a}(\varphi u)^{\prime} v^{\prime} d x d t=0
$$

holds for all $v \in L^{2}\left(0, T ; H^{1,2}(0, a)\right) \cap H^{1,2}\left(0, T ; L^{2}(0, a)\right)$ with $v(T, \cdot)=0$, and the regularity condition

$$
u \varphi \in L^{2}\left(0, T ; H^{1,2}(0, a)\right)
$$

holds.

REMARK 1.2.

1) Since $(u \varphi)^{\prime} \in L^{2}\left(0, T ; L^{2}(0, a)\right)$, the differential equation implies that the distributional derivative $\partial_{t}(u+\varphi) \in L^{2}\left(0, T ; H^{-1.2}(0, a)\right)$ and thus $(u+\varphi) \in C^{0, \frac{1}{2}}([0, T]$; $\left.H^{-1,2}(0, a)\right)$. 
2) We only impose an initial condition for the energy $e=u+\varphi$.

3) The regularity condition substitutes the equilibrium condition as pointed out in the introduction.

4) We still remark that in the solid phase the temperature is ambiguous, if the half-line connecting the point $(t, x)$ in the solid phase and its corresponding initial point $(0, x)$ do not entirely lie in the solid phase.

The main tool in order to prove the convergence of the two-phase to the one-phase problem is an estimate of the total variation of the free boundary of the two-phase problem, which does not depend on the diffusivity coefficients. This estimate in general might not be true for any solution of the two-phase problem, but it is valid for any solution obtained by the time-regularization process as described in the introduction.

DEFinition 1.3. (Regularized two-phase Stefan problem) A pair of functions $u \in$ $L^{2}\left(0, T ; H^{1.2}(0, a)\right) \cap L^{\infty}\left(0, T ; L^{2}(0, a)\right)$ and $s \in H^{1.2}(0, T)$ is a solution of the regularized two-phase Stefan problem with initial data $u_{0} \in L^{2}(0, a)$ and $s_{0} \in[0, a]$ if

$$
\begin{aligned}
& -\int_{0}^{T} \int_{0}^{a} u v_{t} d x d t-\int_{0}^{a} u_{0}(x) v(0, x) d x \\
& \quad+\int_{0}^{T} \int_{0}^{a}\{\delta(1-\varphi)+\varphi\} u^{\prime} v^{\prime} d x d t-\int_{0}^{T} s_{t}(t) v(t, s(t)) d t=0
\end{aligned}
$$

for all $v \in L^{2}\left(0, T ; H^{1.2}(0, a)\right) \cap H^{1,2}\left(0, T ; L^{2}(0, a)\right)$ with $v(T, \cdot)=0$ and where $\varphi(t, x):=$ $H(x-s(t))$;

$0 \leq s(t) \leq a$ for almost all $t$;

for all $\xi \in \mathbb{R}$ and almost all $t \in(0, T):\left(\alpha s_{t}(t)+u(t, s(t))\right) \cdot(\xi-s(t)) \geq 0$; $s(0)=s_{0}$.

REMARK 1.4 .

1) The differential equation together with the regularity of $u$ and $s$ imply that the distributional time derivative $\partial_{t} u$ is a continuous linear functional on $L^{2}\left(0, T ; H_{0}^{1.2}(0, a)\right)$, and thus $\partial_{t} u \in L^{2}\left(0, T ; H^{-1.2}(0, a)\right)$. Hence $u$ has a trace $u(0, \cdot)$ in $H^{-1,2}(0, a)$ at time $t=0$.

2) By choosing $v_{0}(x)\left(1-\frac{t}{\eta}\right)$ as a test function and letting $\eta \rightarrow 0$ we obtain $u(0, \cdot)=u_{0}$.

3) Still note that through the differential equation

$$
\left\|\partial_{t}(u+\varphi)\right\|_{L^{2}\left(0 . T: H^{-1.2}(0 . a)\right)} \leq\left(\int_{0}^{T} \int_{0}^{a}\{\delta(1-\varphi)+\varphi\} u^{\prime 2} d x d t\right)^{1 / 2}
$$

4) The dynamic free boundary condition is formulated in a variational setting in order to take care of the fact that the free boundary may hit the fixed boundary.

The existence of such solutions can be shown following the proof given by Visintin (1987) for the case of equal diffusivity coefficients. Existence of a smooth solution was obtained by Xie (1990) in the case of smooth initial data.

The regularized Stefan problem has a Ljapunov functional which implies natural bounds for the temperature and the free boundary. In addition to this, the total variation of the free boundary $s(t)$ of this regularized two-phase problem satisfies a bound, which is uniform in both the regularization parameter $\alpha$ and the heat diffusivity coefficient $\delta$. 
Proposition 1.5. Any solution $(u, s)$ of the regularized two-phase Stefan problem satisfies the energy estimate

$$
\sup _{t} \int_{0}^{a} u^{2}(t, x) d x+\int_{0}^{T} \int_{0}^{a}\{\delta(1-\varphi)+\varphi\}\left(u^{\prime}\right)^{2} d x d t+\alpha \int_{0}^{T}\left|s_{t}(t)\right| d t \leq \int_{0}^{a} u_{0}^{2} d x
$$

and the BV-bound

$$
\int_{0}^{T}\left|s_{t}(t)\right| d t \leq \int_{0}^{a}\left|u_{0}\right| d x
$$

We will give the proof of this central proposition in Sec. 2 .

Let us now come back to the two-phase Stefan problem. The definition for a solution is very similar to that of the regularized problem. The basic changes are due to the fact that the free boundary no longer will be of class $H^{1,2}$ and thus the initial condition for the free boundary has to be formulated within the differential equation.

Definition 1.6. (Two-phase Stefan problem) A pair of functions $u \in L^{\infty}(0, T$; $\left.L^{2}(0, a)\right)$ and $s \in \mathrm{BV}(0, T)$ is a solution of the two-phase Stefan problem with initial data $u_{0} \in L^{2}(0, a)$ and $s_{0} \in[0, a]$ if for the order parameter

$$
\varphi(t, x):=H(x-s(t)) \text { and } \varphi_{0}(x):=H\left(x-s_{0}\right)
$$

and the temperature $u$, the differential equation

$$
\begin{gathered}
-\int_{0}^{T} \int_{0}^{a}(u+\varphi) v_{t} d x d t-\int_{0}^{a}\left(u_{0}+\varphi_{0}\right)(x) v(0, x) d x \\
+\int_{0}^{T} \int_{0}^{a}\{\delta(1-\varphi)+\varphi\} u^{\prime} v^{\prime} d x d t=0
\end{gathered}
$$

holds for all $v \in L^{2}\left(0, T ; H^{1,2}(0, a)\right) \cap H^{1,2}\left(0, T ; L^{2}(0, a)\right)$ with $v(T, \cdot)=0$;

$0 \leq s(t) \leq a$ for almost all $t$;

for all $\xi \in \mathbb{R}$ and almost all $t \in(0, T): u(t, s(t)) \cdot(\xi-s(t)) \geq 0$.

Now, for any $\delta>0$ there exists a solution of the two-phase problem that can be obtained as the limit of a sequence of solutions of the regularized problem. This result has been shown by Visintin for the case of equal heat diffusivity coefficients (1987), and still holds true here. One important feature of this solution is that the bound on the total variation of the free boundary, as obtained in Proposition 1.5, is independent of both $\alpha$ and $\delta$. This is the content of

Proposition 1.7. For any $\delta>0$ and any initial data $u_{\delta 0} \in L^{2}(0, a)$ and $s_{\delta 0} \in[0, a]$ there exists a solution $\left(u_{\delta}, s_{\delta}\right)$ of the two-phase Stefan problem, which can be approximated by a sequence $\left(u_{\delta \alpha}, s_{\delta \alpha}\right)$ of solutions of the regularized Stefan problem in the following sense:

$$
\begin{array}{rlrl}
\dot{s}_{\delta \alpha} & \stackrel{*}{\rightarrow} \dot{s}_{\delta} & & \text { in the weak-*-topology of }\left[C^{0}(0, T)\right]^{*}, \\
s_{\delta \alpha} & \rightarrow s_{\delta} & \text { strongly in } L^{p}(0, T) \text { for any } 1 \leq p<\infty, \\
u_{\delta \alpha}, u_{\delta \alpha}^{\prime} & \rightarrow u_{\delta}, u_{\delta}^{\prime} & \text { weakly in } L^{2}((0, T) \times(0, a)), \\
\partial_{t} \varphi_{\delta \alpha} & \stackrel{*}{\rightarrow} \partial_{t} \varphi_{\delta} & & \text { in the weak-*-topology of }\left[C^{0}((0, T) \times(0, a))\right]^{*}, \\
\varphi_{\delta \alpha} & \rightarrow \varphi_{\delta} & \text { strongly in } L^{p}((0, T) \times(0, a)) .
\end{array}
$$


In addition, we have the estimates

$$
\sup _{t} \int_{0}^{a} u_{\delta}^{2}(t, x) d x+\int_{0}^{T} \int_{0}^{a}\left\{\delta\left(1-\varphi_{\delta}\right)+\varphi_{\delta}\right\}\left(u_{\delta}^{\prime}\right)^{2} d x d t \leq \int_{0}^{a} u_{\delta 0}^{2} d x
$$

and

$$
\int_{0}^{T}\left|\left(s_{\delta}\right)_{t}(t)\right| d t \leq \int_{0}^{a}\left|u_{\delta 0}\right| d x
$$

REMARK 1.8. Proposition 1.5 implies that for $u=u_{\delta}$ as above

$$
\int_{0}^{a} u^{2}(t, x) d x \leq \int_{0}^{a} u_{0}^{2}(x) d x
$$

for almost all $t>0$. Assume that there is an initial jump of the interface, but that apart from that the solution is nice:

$$
\lim _{t \rightarrow 0} s(t)=: s_{+}<s_{0}
$$

Since $u+\varphi$ has to be continuous in time, the temperature has to compensate for the jump in $\varphi$ :

$$
\lim _{t \rightarrow 0} u(t, x):=u_{+}(x)= \begin{cases}u_{0}(x)-1, & \text { if } x \in\left(s_{+}, s_{0}\right) \\ u_{0}(x), & \text { else. }\end{cases}
$$

Using the monotonicity of the $L^{2}$-norm, we find

$$
\int_{0}^{a} u_{+}^{2}(x)-u_{0}^{2}(x) d x=\int_{s+}^{s_{0}}\left(-2 u_{0}(x)+1\right) d x \leq 0
$$

implying

$$
s_{0}-s_{+} \leq 2 \int_{s_{+}}^{s_{0}} u_{0}(x) d x
$$

We conclude that in order to have an initial jump, a "sufficient" amount of overheating is necessary. For more detailed discussions of these questions we refer to Gurtin (1992) and Götz and Zaltzman (1992).

Proof of Proposition 1.7. We denote by $\left(u_{\delta_{\alpha}}, s_{\delta_{\alpha}}\right)$ a solution of the regularized problem with initial conditions $u_{\delta(0)}$ and $s_{\delta()}$. Note that any solution of the regularized Stefan problem satisfies as well the differential equation as imposed for the nonregularized problem. Then the bounds of Proposition 1.5 allow to subtract a subsequence $\alpha \rightarrow 0$ that converges as claimed. This convergence then immediately implies that the differential equation is satisfied for the limit. To show the convergence of the free boundary equation, we first observe that $\alpha\left(s_{\delta_{\alpha}}\right)_{t}$ converges to zero in $L^{1}(0, T)$, due to the bound for $\left(s_{\delta \alpha}\right)_{t}$ of Proposition 1.5. So we only have to prove that $u_{\delta \alpha}\left(t, s_{\delta \alpha}(t)\right)$ converges to $u_{\delta}\left(t, s_{\delta}(t)\right)$. By Proposition $1.5\left(u_{\delta \alpha}\right)^{\prime}$ is in $L^{2}$ uniformly in $\alpha$ and according to Remark $1.4(3)$ the time derivative $\partial_{t}\left(u_{\delta \alpha}+\varphi_{\delta \alpha}\right)$ is bounded in $L^{2}\left(0, T ; H^{-1,2}(0, a)\right)$ uniformly in $\alpha$ and, by construction, $\varphi_{\delta \alpha} \in L^{\infty}(0, T ; \operatorname{BV}(0, a))$ with values in $\{0,1\}$. Thus a lemma of Luckhaus 
applies [Luckhaus 1990, Lemma 2], giving a bound on fractional time derivatives of $u_{\delta \alpha}$ and $\varphi_{\delta \alpha}$ :

$$
\begin{aligned}
& \int_{\tau}^{T} \int_{0}^{a}\left|\varphi_{\delta \alpha}(t, x)-\varphi_{\delta \alpha}(t-\tau, x)\right| d x d t \\
& \quad+\int_{\tau}^{T} \int_{0}^{a}\left|u_{\delta \alpha}(t, x)-u_{\delta \alpha}(t-\tau, x)\right| d x d t \leq C(\delta) \tau^{\frac{1}{\delta}}
\end{aligned}
$$

with a constant independent of $\alpha$ (but depending on $\delta$ through the $L^{2}$-norm of the temperature gradient). This implies that we can select a further subsequence such that

$$
u_{\delta \alpha} \rightarrow u_{\delta} \quad \text { in } L^{1}((0, T) \times(0, a))
$$

Now we estimate

$$
\begin{aligned}
& \int_{0}^{T}\left|u_{\delta \alpha}\left(t, s_{\delta \alpha}(t)\right)-u_{\delta}\left(t, s_{\delta}(t)\right)\right| d t \\
& \leq\left(\int_{0}^{T} \int_{0}^{a}\left(u_{\delta \alpha}^{\prime}\right)^{2} d x d t\right)^{1 / 2}\left(\int_{0}^{T}\left|s_{\delta \alpha}-s_{\delta}\right| d t\right)^{1 / 2}+\frac{1}{\mu} \int_{0}^{t} \int_{s(t)}^{s(t)+\mu}\left|u_{\delta \alpha}-u_{\delta}\right| d x d t \\
& \quad+2 \mu^{1 / 2} T^{1 / 2}\left(\int_{0}^{T} \int_{0}^{a}\left(u_{\delta \alpha}^{\prime}\right)^{2} d x d t\right)^{1 / 2} \\
& \leq C(\delta)\left(\int_{0}^{T}\left|s_{\delta \alpha}-s_{\delta}\right| d t+\mu^{1 / 2}\right)^{1 / 2}+\frac{1}{\mu} \int_{0}^{T} \int_{0}^{a}\left|u_{\delta \alpha}-u_{\delta}\right| d x d t
\end{aligned}
$$

Since $\mu$ can be chosen arbitrarily small this implies that

$$
u_{\delta \alpha}\left(\cdot, s_{\delta \alpha}\right) \rightarrow u_{\delta}\left(\cdot, s_{\delta}\right) \quad \text { in } L^{1}(0, T)
$$

Thus we find for all $\xi \in \mathbb{R}$ and almost all $t \in(0, T)$ :

$$
u_{\delta}\left(t, s_{\delta}(t)\right) \cdot\left(\xi-s_{\delta}(t)\right) \geq 0
$$

This proposition enables us now to show that, as $\delta \rightarrow 0$, a solution of the one-phase problem is obtained.

TheOrem 1.9. Assume that $u_{\delta 0} \rightarrow u_{0}$ weakly in $L^{2}(0, a)$ and $s_{\delta 0} \rightarrow s_{0}$. Then a subsequence of the solutions of the two-phase problem as constructed in Prop. 1.7 converges to a solution of the one-phase Stefan problem.

Proof. By Proposition 1.7. we may select a subsequence $\delta \rightarrow 0$ such that

$$
\begin{array}{ll}
\dot{s}_{\delta} \stackrel{*}{\rightarrow} \dot{s} & \text { in the weak-*-topology of }\left[C_{0}^{0}(0, T)\right]^{*}, \\
s_{\delta} \rightarrow s & \text { strongly in } L^{p}(0, T) \text { for any } 1 \leq p<\infty, \\
u_{\delta} \rightarrow u & \text { weakly in } L^{2}((0, T) \times(0, a)),
\end{array}
$$


and

$$
\begin{aligned}
& \partial_{t} \varphi_{\delta} \stackrel{*}{\rightarrow} \partial_{t} \varphi \quad \text { in the weak-*-topology of }\left[C^{0}((0, T) \times(0, a))\right]^{*}, \\
& \varphi_{\delta} \rightarrow \varphi \quad \text { strongly in } L^{p}((0, T) \times(0, a)) \text {. }
\end{aligned}
$$

Since $u_{\delta}(t, \cdot)=0$ on supp $\varphi_{\delta}^{\prime}$, we have

$$
\left(\varphi_{\delta} u_{\delta}\right)^{\prime}=\varphi_{\delta} u_{\delta}^{\prime} .
$$

This implies that

$$
\begin{aligned}
\int_{0}^{T} \int_{0}^{a}\left|\left(\varphi_{\delta} u_{\delta}\right)^{\prime}\right|^{2} d x d t & =\int_{0}^{T} \int_{0}^{a} \varphi_{\delta}\left|u_{\delta}^{\prime}\right|^{2} d x d t \\
& \leq \int_{0}^{T} \int_{0}^{a}\left\{\delta\left(1-\varphi_{\delta}\right)+\varphi_{\delta}\right\}\left|u_{\delta}^{\prime}\right|^{2} d x d t \leq C
\end{aligned}
$$

with a constant independent of $\delta$. Thus for some further subsequence

$$
\left(\varphi_{\delta} u_{\delta}\right)^{\prime} \rightarrow w \text { weakly in } L^{2}((0, T) \times(0, a)) .
$$

In addition, we know that

$$
\varphi_{\delta} u_{\delta} \rightarrow \varphi u \quad \text { weakly in } L^{2}((0, T) \times(0, a)) .
$$

This implies that

$$
(\varphi u)^{\prime} \in L^{2}((0, T) \times(0, a))
$$

and

$$
\left(\varphi_{\delta} u_{\delta}\right)^{\prime} \rightarrow(\varphi u)^{\prime} \quad \text { weakly in } L^{2}((0, T) \times(0, a)) .
$$

This allows us to pass to the limit in the differential equation of the two-phase problem. In particular,

$$
\begin{aligned}
\int_{0}^{T} \int_{0}^{a}\left\{\delta\left(1-\varphi_{\delta}\right)+\varphi_{\delta}\right\} u_{\delta}^{\prime} v^{\prime} d x d t= & \delta \int_{0}^{T} \int_{0}^{a}\left(1-\varphi_{\delta}\right) u_{\delta}^{\prime} v^{\prime} d x d t \\
& +\int_{0}^{T} \int_{0}^{a}\left(\varphi_{\delta} u_{\delta}^{\prime}\right) v^{\prime} d x d t \\
\rightarrow 0 & +\int_{0}^{T} \int_{0}^{a}\left(\varphi u^{\prime}\right) v^{\prime} d x d t
\end{aligned}
$$

because

$$
\begin{aligned}
& \left|\delta \int_{0}^{T} \int_{0}^{a}\left(1-\varphi_{\delta}\right) u_{\delta}^{\prime} v^{\prime} d x d t\right| \\
& \quad \leq \delta^{1 / 2}\left(\int_{0}^{T} \int_{0}^{a}\left(1-\varphi_{\delta}\right) u_{\delta}^{\prime 2} d x d t\right)^{1 / 2}\left(\int_{0}^{T} \int_{0}^{a} v^{\prime 2} d x d t\right)^{1 / 2} \leq C \delta^{1 / 2} .
\end{aligned}
$$

This proves the theorem.

We still note that $(\varphi u)^{\prime} \in L^{2}((0, T) \times(0, a))$ implies that

$$
u(t, x) \rightarrow 0 \quad \text { as } x \text { approaches } s(t) \text { from above. }
$$

In addition, $u^{\prime} \in L^{2}(\{(t, x): x>s(t)\})$ and

$$
(\varphi u)^{\prime}=\varphi u^{\prime}
$$


Section 2. Proof of Proposition 1.5. This section deals with the essential mathematics of our problem, the proof of the a priori estimates of Proposition 1.5. The first claim of the proposition may be seen as a consequence of the existence of a natural Ljapunov functional. Indeed, it may formally be obtained by just choosing $u$ as a test function. To obtain the second estimate the best choice is $\operatorname{sgn}(u)$ as a test function. But of course, in particular, the second test function has by no means the required regularity, such that we have to construct suitable approximations, thereby recovering the structure of the desired test functions and taking care of the initial and terminal values. We want to treat both estimates at the same time. So let

$$
f: \mathbb{R} \rightarrow \mathbb{R}
$$

be a Lipschitz function (later either the identity or an approximation of the sign-function) and extend $u$ by its initial values to negative values of $t$. Then set

$$
v_{\varepsilon}:=f\left((\eta u) * \psi_{\varepsilon}\right)
$$

where $\psi_{\varepsilon}$ is a symmetric, smooth Dirac sequence with compact support, only depending on $t$, and $\eta$ is a cut-off function in time:

$$
\begin{aligned}
\eta(t)=1, & \text { if } 0 \leq t \leq t_{0} \\
\eta(t)=0, & \text { if } t \leq-\gamma \text { or } t_{0}+\gamma \leq t \\
\eta^{\prime}(t)=\frac{1}{\gamma}, & \text { if }-\gamma \leq t \leq 0 \\
\eta^{\prime}(\dot{\imath})=-\frac{1}{\gamma}, & \text { if } t_{0} \leq t \leq t_{0}+\gamma
\end{aligned}
$$

In order to keep the structure we choose

$$
\left(v_{\varepsilon} * \psi_{\varepsilon}\right) \cdot \eta
$$

as a test function. If $t_{0}<T$ this is an admissible test function, satisfying the regularity requirements in the variable $x$ by the definition of $u$ and in the variable $t$ by smoothening through $\psi_{\varepsilon}$, and the vanishing condition at $T$ through the cut-off. Thus,

$$
\begin{aligned}
& -\int_{0}^{T} \int_{0}^{a} u\left[\left(v_{\varepsilon} * \psi_{\varepsilon}\right) \cdot \eta\right]_{t} d x d t-\int_{0}^{a} u_{0}\left(v_{\varepsilon} * \psi_{\varepsilon}\right)(0, x) d x \\
& \quad+\int_{0}^{T} \int_{0}^{a}\{\delta(1-\varphi)+\varphi\} u^{\prime}\left[\left(v_{\varepsilon} * \psi_{\varepsilon}\right) \cdot \eta\right]^{\prime} d x d t-\int_{0}^{T} s_{t}(t)\left[\left(v_{\varepsilon} * \psi_{\varepsilon}\right) \cdot \eta\right](t, s(t)) d t=0 .
\end{aligned}
$$


Let us calculate the first term:

$$
\begin{aligned}
-\int_{0}^{T} \int_{0}^{a} u\left[\left(v_{\varepsilon} * \psi_{\varepsilon}\right) \cdot \eta\right]_{t} d x d t \\
=-\int_{0}^{T} \int_{0}^{a} u \eta\left(v_{\varepsilon}\right)_{t} * \psi_{\varepsilon} d x d t-\int_{0}^{T} \int_{0}^{a} u\left(v_{\varepsilon} * \psi_{\varepsilon}\right) \eta_{t} d x d t \\
=-\int_{-\infty}^{\infty} \int_{0}^{a} u \eta\left(v_{\varepsilon}\right)_{t} * \psi_{\varepsilon} d x d t+\int_{-\gamma}^{0} \int_{0}^{a} u_{0} \eta\left(v_{\varepsilon} * \psi_{\varepsilon}\right)_{t} d x d t \\
+\frac{1}{\gamma} \int_{t_{0}}^{t_{0}+\gamma} \int_{0}^{a} u\left(v_{\varepsilon} * \psi_{\varepsilon}\right) d x d t \\
=-\int_{-\infty}^{\infty} \int_{0}^{a}\left[(u \eta) * \psi_{\varepsilon}\right]\left(v_{\varepsilon}\right)_{t} d x d t-\frac{1}{\gamma} \int_{-\gamma}^{0} \int_{0}^{a} u_{0}\left(v_{\varepsilon} * \psi_{\varepsilon}\right) d x d t \\
+\int_{0}^{a} u_{0} \eta\left(v_{\varepsilon} * \psi_{\varepsilon}\right)(0, x) d x d t+\frac{1}{\gamma} \int_{t_{0}}^{t_{0}+\gamma} \int_{0}^{a} u\left(v_{\varepsilon} * \psi_{\varepsilon}\right) d x d t \\
=\int_{-\infty}^{\infty} \int_{0}^{a} \partial_{t} F\left((u \eta) * \psi_{\varepsilon}\right) d x d t-\frac{1}{\gamma} \int_{-\gamma}^{0} \int_{0}^{a} u_{0}\left(v_{\varepsilon} * \psi_{\varepsilon}\right) d x d t \\
+\int_{0}^{a} u_{0} \eta\left(v_{\varepsilon} * \psi_{\varepsilon}\right)(0, x) d x+\frac{1}{\gamma} \int_{t_{0}}^{t_{0}+\gamma} \int_{0}^{a} u\left(v_{\varepsilon} * \psi_{\varepsilon}\right) d x d t
\end{aligned}
$$

Here $F$ is a primitive of $f$ with $F(0)=0$, and since $v_{\varepsilon}$ vanishes at $\pm \infty$ the $F$-term is 0 . We thus arrive at the following formula for the first two terms of $(*)$ :

$$
(1 *)+(2 *)=-\frac{1}{\gamma} \int_{-\gamma}^{0} \int_{0}^{a} u_{0}\left(v_{\varepsilon} * \psi_{\varepsilon}\right) d x d t+\frac{1}{\gamma} \int_{t_{0}}^{t_{0}+\gamma} \int_{0}^{a} u\left(v_{\varepsilon} * \psi_{\varepsilon}\right) d x d t
$$

By construction we know that

$$
(u \eta) * \psi_{\varepsilon} \stackrel{\varepsilon \rightarrow 0}{\rightarrow} u \eta \quad \text { in } L^{2}((0, T) \times(0, a)),
$$

and since $f$ is Lipschitz, both

$$
v_{\varepsilon} \text { and } v_{\varepsilon} * \psi_{\varepsilon} \stackrel{\varepsilon \rightarrow 0}{\rightarrow} v:=f(u \eta) \quad \text { in } L^{2}((0, T) \times(0, a))
$$

as well. So we conclude

$$
(1 *)+(2 *) \stackrel{\varsigma \rightarrow 0}{\rightarrow}-\frac{1}{\gamma} \int_{-\gamma}^{0} \int_{0}^{a} u_{0} v d x d t+\frac{1}{\gamma} \int_{t_{0}}^{t_{0}+\gamma} \int_{0}^{a} u v d x d t
$$

We next observe that $\left(v_{\varepsilon} * \psi_{\varepsilon}\right)^{\prime}$ is uniformly bounded in $L^{2}((0, T) \times(0, a))$, such that

$$
\left(v_{\varepsilon} * \psi_{\varepsilon}\right)^{\prime} \stackrel{\varepsilon \rightarrow 0}{\rightarrow} v^{\prime} \quad \text { in } L^{2}((0, T) \times(0, a))
$$

and

$$
\left(v_{\varepsilon} * \psi_{\varepsilon}\right)(\cdot, s) \stackrel{\varepsilon \rightarrow 0}{\rightarrow} v(\cdot, s) \quad \text { in } L^{2}(0, T)
$$


Thus, the third and the fourth terms in $(*)$ converge to the respective terms where $v_{\varepsilon} * \psi_{\varepsilon}$ is substituted by $v$. Altogether we find

$$
\begin{aligned}
& -\frac{1}{\gamma} \int_{-\gamma}^{0} \int_{0}^{a} u_{0} v d x d t+\frac{1}{\gamma} \int_{t_{0}}^{t_{0}+\gamma} \int_{0}^{a} u v d x d t \\
& \quad+\int_{0}^{T} \int_{0}^{a}\{\delta(1-\varphi)+\varphi\} u^{\prime} v^{\prime} \eta d x d t-\int_{0}^{T} s_{t}(t) v(t, s(t)) \eta(t) d t=0
\end{aligned}
$$

Now choose $f(\lambda)=\lambda$, so that $v=u \eta$. Then, as $\gamma \rightarrow 0$,

$$
-\frac{1}{\gamma} \int_{-\gamma}^{0} \int_{0}^{a} u_{0} v d x d t+\frac{1}{\gamma} \int_{t_{0}}^{t_{0}+\gamma} \int_{0}^{a} u v d x d t \rightarrow-\frac{1}{2} \int_{0}^{a} u_{0}^{2} d x+\frac{1}{2} \int_{0}^{a} u\left(t_{0}, x\right) d x
$$

for almost every $t_{0}$ and

$$
\begin{aligned}
\int_{0}^{T} \int_{0}^{a}\{ & \delta(1-\varphi)+\varphi\} u^{\prime} v^{\prime} \eta d x d t-\int_{0}^{T} s_{t}(t) v(t, s(t)) \eta(t) d t \\
& \rightarrow \int_{0}^{t_{0}} \int_{0}^{a}\{\delta(1-\varphi)+\varphi\} u^{\prime 2} d x d t-\int_{0}^{t_{0}} s_{t}(t) v(t, s(t)) d t
\end{aligned}
$$

But almost everywhere in $\{s \neq 0, a\}$ the dynamic free boundary condition implies $u(t, s(t))=-\alpha s_{t}(t)$, and almost everywhere in $\{s=0, a\}$ the time derivative vanishes, such that $-s_{t}(t) u(t, s(t))=\alpha s_{t}^{2}(t)$ and thus the first estimate of the Proposition is shown.

In order to prove the second we take

$$
f(\lambda)=f_{n}(\lambda):= \begin{cases}-1, & \text { if } \lambda \leq-\frac{1}{n} \\ n \lambda, & \text { if }-\frac{1}{n} \leq \lambda \leq \frac{1}{n} \\ 1, & \text { if } \frac{1}{n} \leq \lambda\end{cases}
$$

which is a standard Lipschitz approximation of the sign-function. In this case $v=f_{n}(u \eta)$. Since $f_{n}$ is sign preserving we have

$$
\frac{1}{\gamma} \int_{t_{0}}^{t_{0}+\gamma} \int_{0}^{a} u v d x d t \geq 0
$$

and, since $f_{n}$ is bounded by 1 ,

$$
\frac{1}{\gamma} \int_{-\gamma}^{0} \int_{0}^{a} u_{0} v d x d t \leq \int_{0}^{a}\left|u_{0}\right| d x
$$

Using the monotonicity of $f_{n}$ gives $u^{\prime} v^{\prime}$ positive, such that $(* *)$ implies

$$
-\int_{0}^{T} s_{t} f_{n}(u(\cdot, s) \eta) \eta d t \leq \int_{0}^{a}\left|u_{0}\right| d x
$$

But as above either $s_{t}=0$ or $u(t, s(t))=-\alpha s_{t}(t)$. Substituting this and letting first $n$, then $\gamma$ converge to zero establishes the second estimate of Proposition 1.5. The proof is thus complete. 
Section 3. The radial problem. In the first two sections we focussed on the onedimensional problem and thus neglected the effects of curvature. Here we will briefly outline that the same method as presented in the first two sections works for the radial problem in higher dimensions. The basic changes are to be made concerning the regularized two-phase problem. The curvature term will be incorporated in the dynamical free boundary condition, which is known as the Gibbs-Thomson relation. We follow again the approach of Visintin by defining the spaces:

$$
\begin{aligned}
W & :=\left\{v:(0, a) \text { measurable } \rightarrow \mathbb{R}: \int_{0}^{a}|v(x)|^{2} x^{N-1} d x<\infty\right\}, \\
V & :=\left\{v \in W: v^{\prime} \in W\right\} \\
Z & :=V \cap C^{0}([0, a]) .
\end{aligned}
$$

Let $u_{0} \in W$ and $s_{0} \in[0, a]$.

Definition And Proposition 3.1. (Radial two-phase Stefan problem with dynamical Gibbs-Thomson relation) There exists a $u \in L^{2}(0, T ; V) \cap L^{\infty}(0, T ; W) \cap H^{1.2}\left(0, T ; Z^{\prime}\right)$ and a free boundary $s \in L^{\infty}(0, T)$ such that $s^{N} \in H^{1.2}(0, T)$, which are solutions of the free boundary value problem:

$$
\begin{aligned}
u(t, s(t)) \cdot s(t)^{N-1} \in L^{1}(0, T) & ; \\
-\int_{0}^{T} \int_{0}^{a} u \dot{v} x^{N-1} d x d t & +\int_{0}^{T} \int_{0}^{a}\{(1-\varphi)+\delta \varphi\} u^{\prime} v^{\prime} x^{N-1} d x d t \\
& +\frac{1}{N} \int_{0}^{T} \frac{d}{d t}\left[s(t)^{N}\right] v(t, s(t)) d t=0
\end{aligned}
$$

for all $v \in L^{2}(0, T ; Z) \cap H^{1.2}(0, T ; W)$ such that $v(0, \cdot)=v(T, \cdot)=0$;

$0 \leq s(t) \leq a$ almost everywhere in $(0, T)$;

$\left\{\alpha\left(\frac{1}{N} \frac{d}{d t}\left[s(t)^{N}\right]+s(t)^{N-2}\right)-u(t, s(t)) \cdot s(t)^{N-1}\right\} \cdot[\xi-s(t)] \geq 0$ for all $\xi \in \mathbb{R} ;$

$u(0, \cdot)=u_{0}$ in $Z^{\prime}$;

$s(0)=s_{0}$.

The crucial step now is to obtain again the bounds as in Proposition 1.5, in particular, the estimate on the total variation of the free boundary. The first estimate of Proposition 1.5 still holds true in the radial setting, since it was a consequence of the existence of a Ljapunov functional.

Proposition 3.2. Any solution of the two-phase Stefan problem as in Definition 3.1 satisfies

$$
\int_{0}^{T} \frac{1}{N}\left|\frac{d}{d t} s^{N}(t)\right| d t \leq 2 a^{N-2} T+\int_{0}^{a}\left|u_{0}(x)\right| x^{N-1} d x .
$$

Proof. Following the same strategy as in Sec. 2 we find

$$
\int_{0}^{T} \frac{1}{N} \frac{d}{d t} s^{N}(t) \operatorname{sgn} u(t, s(t)) d t \leq \int_{0}^{a}\left|u_{0}(x)\right| x^{N-1} d x .
$$


Now we observe that either $\operatorname{sgn} u(t, s(t))=\operatorname{sgn} \dot{s}(t)$ or $\dot{s}(t)$ is bounded: If $u(t, s(t)) \neq 0$ and $s(t) \neq 0$ or $a$, then the free boundary condition implies

$$
\alpha\left(\frac{1}{N} \frac{d}{d t} s^{N}(t)+s^{N-2}(t)\right)=u(t, s(t)) s^{N-1}(t)
$$

and thus

$$
\operatorname{sgn} u(t, s(t))=\operatorname{sgn}\left(\frac{1}{N} \frac{d}{d t} s^{N}(t)+s^{N-2}(t)\right) .
$$

If either $\frac{1}{N} \frac{d}{d t} s^{N}(t)>0$ or $\frac{1}{N} \frac{d}{d t} s^{N}(t)<-s^{N-2}(t)$, then clearly

$$
\operatorname{sgn}\left(\frac{1}{N} \frac{d}{d t} s^{N}(t)+s^{N-2}(t)\right)=\operatorname{sgn}\left(\frac{1}{N} \frac{d}{d t} s^{N}(t)\right)
$$

and otherwise

$$
\left|\frac{1}{N} \frac{d}{d t} s^{N}(t)\right| \leq s^{N-2}(t) .
$$

The last inequality holds as well in the case when $u(t, s(t))=0$, and in the set $\{s(t)=a\}$ and $\{s(t)=0\}$ the time derivative vanishes almost everywhere. Putting all this together we conclude that

$$
\begin{aligned}
\int_{0}^{T} \frac{1}{N}\left|\frac{d}{d t} s^{N}(t)\right| d t & \leq 2 \int_{0}^{T} s^{N-2}(t) d t+\int_{0}^{a}\left|u_{0}\right|(x) x^{N-1} d x \\
& \leq 2 a^{N-2} T+\int_{0}^{a}\left|u_{0}\right|(x) x^{N-1} d x
\end{aligned}
$$

This estimate does neither depend on the parameter $\alpha$ nor on $\delta$.

Once this Proposition is obtained we proceed as in Sec. 1. Thus, we first obtain a solution of the two-phase Stefan problem through the regularization process, and then a solution of the one-phase problem. In particular, Proposition 3.2 ensures that the order parameter

$$
\varphi_{\delta \alpha}(t, x)=H\left(x-s_{\delta \alpha}(t)\right)
$$

converges pointwise almost everywhere as first the time regularization parameter $\alpha$ and then the diffusivity coefficient $\delta$ converge to zero. The limit then has the same structure and is given by some

$$
\varphi(t, x)=H(x-s(t)),
$$

where $s$ defines the free boundary of the limit one-phase Stefan problem.

The definitions of the two-phase and the one-phase Stefan problem follow the same spirit as those for the one-dimensional case and we do not give them explicitly. Once again the equilibrium condition for the one-phase problem will be given by

$$
u \varphi \in L^{2}(0, T ; V)
$$

Acknowledgment. The author likes to thank Morton Gurtin for drawing her attention to this problem, and to Stefan Müller for valuable discussions. 


\section{REFERENCES}

[1] I. G. Götz and B. Zaltzman, Two-Phase Stefan Problem with Supercooling, preprint (1993)

[2] M. E. Gurtin, Thermodynamics and the Supercritical Stefan Equations with Nucleations, CMUreport (1992)

[3] St. Luckhaus, Solutions of the two phase Stefan problem with the Gibbs-Thomson relation for the melting temperature, Europ. J. Appl. Math. 1, 101-111 (1990)

[4] A. Visintin, Stefan problem with a kinetic condition at the free boundary, Annali Mat. Pura Appl. 146, $97-122(1987)$

[5] W. Xie, The Stefan problem with a kinetic condition at the free boundary, SIAM J. Math. Anal. 21, no. 2, 362-373 (1990) 